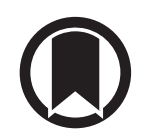

CrossMark

\title{
Careful consideration of the bleeding caused by transbronchial lung cryobiopsies
}

\author{
To the Editor:
}

We read with great interest a research letter by PANNU et al. [1] in the European Respiratory Journal describing unexpected high mortality results in association with transbronchial lung cryobiopsy (TBLC). The team at Vanderbilt University Medical Center has decided to no longer provide TBLC for inpatients with acute diffuse parenchymal lung disease (DPLD) exacerbations, and spotlighted bleeding complications as a predictive factor for a higher risk of mortality for all patients [1].

Bleeding, together with pneumothorax, is one of the most commonly reported complications in association with TBLC [2]. Cryobiopsy is clearly capable of causing massive and severe haemorrhage, as reported in the review article by LENTZ et al. [3], with $12 \%$ of patients experiencing serious haemorrhage immediately after cryobiopsy, including one patient who survived a life-threatening bleeding at an American academic medical centre [4].

The recent CryoPID [5] and COLDICE [6] studies performed traditional surgical lung biopsy (SLB) immediately subsequent to TBLC, with the goal of comparing pathology results between the two biopsy techniques. The immediate performance of thoracovideoscopy for SLB just after TBLC provided an opportunity to photograph the wounds and haematomas inflicted by TBLC. When removing a TBLC sample from the lungs, tearing of the capillary beds around the frozen chunk of tissue creates ideal conditions for haemorrhage. As demonstrated in figure 1, TBLC can cause quite an extensive lung parenchyma wound and intra-pleural haemorrhage. As further demonstrated in figure 1c, haematomas can be extensive and haemorrhage can be either intra-parenchymatous, where it can be seen progressing "live" with fluoroscopy, or intra-pleural.

How much parenchyma is at least temporarily blocked by blood awaiting reabsorption is a pertinent question. The adverse event "bleeding" in TBLC cases may not be just about blood loss, but also about a loss of lung capacity. We were thus quite pleased to see the prophylactic assessment protocol proposed by PANNU et al. [1]. The latter test the efficacy of bleeding blockades and potential decreases in residual lung function prior to proceeding with TBLC in case of complications. This provides a logical and objective opportunity for switching to SLB when the potential consequences of common TLBC complications are deemed too severe. On the other hand, it further complicates an already complex procedure, and this is likely to weigh in favour of SLB. It would be interesting to develop further data describing how many potential TBLC patients would be converted to SLB based on failure of these tests, as well as associated effects on mortality rates.

In summary, despite being less invasive in nature, the risk of bleeding complications during TBLC still requires careful consideration. "Soiling of the airways" by blood [1] can have dangerous consequences for DLPD populations with potential consequences for overall survival [1]. The observed risk of TBLC diagnostic failure in terms of histopathology was reported as 6/65 (9.23\%) individuals in the COLDICE [6] study and 4/21 (19.0\%) individuals in the CryoPID [5] study (a pooled total of $10.7 \pm 3.3 \%$ via unpublished meta-analysis). The COLDICE study further evidenced that 11/65 (16.9\%) of TBLCs were indeterminate for usual interstitial pneumonia [6]. Taking this into account in the current situation, where TBLC has entered routine practice in some centres, we support the creation of transparent registries that include the exhaustive recording of safety issues and/or ethically approved clinical trials. As the large majority of TBLC procedures successfully terminate free of adverse events, it is likely that we can take advantage of

@ERSpublications

Lung surface photos show wounds/haemorrhages caused by transbronchial lung cryobiopsy (TBLC). TBLC bleeding can extensively soil airways with consequences for lung function, stressing the need for bleeding prevention and more research via registries. https://bit.ly/34wWAeR

Cite this article as: Bourdin A, Romagnoli M, Gamez AS, et al. Careful consideration of the bleeding caused by transbronchial lung cryobiopsies. Eur Respir J 2020; 55: 1902415 [https://doi.org/10.1183/ 13993003.02415-2019]. 

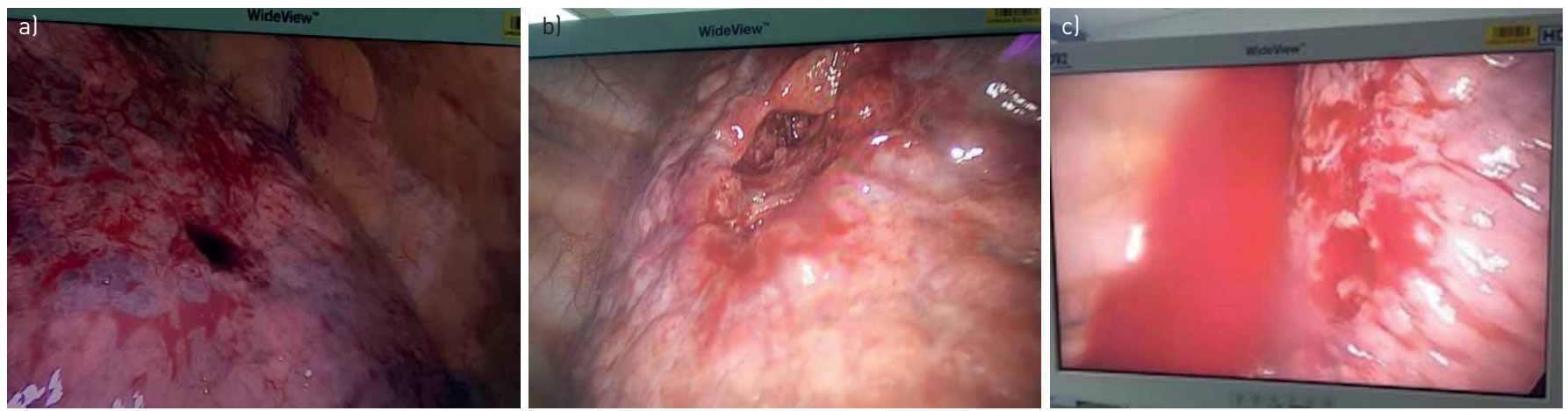

FIGURE 1 Three snapshots of the surface of the lung as it appeared on the monitor during video thoracoscopy for surgical lung biopsy immediately following transbronchial lung cryobiopsy (TBLC) (published with the patient's permission). If the bronchoscopist has good peripheral technique (i.e. he/she can reach sub-pleural parenchyma tissues), the resulting TBLC wound is obvious upon video thoracoscopy (a), and again in panel after suctioning (b). The spread of the associated haematoma to surrounding tissues can be visibly extensive and raises concern for a corresponding decrease in lung capacity. Bleeding can also be pleural in nature (c).

the diagnostic yield if standardisation and appropriate patient selection in experienced centres are secured. Of note, multiple confounding factors may affect the benefit/risk ratio (including the risk of death) of any procedure and this should be taken into account. In COLDICE [6] and CryoPID [5], TBLCs were performed using the same exclusion criteria as those consensually acknowledged for SLB, and we recommend not going beyond these points (very low diffusing capacity of the lung for carbon monoxide, fast declining or acute interstitial lung disease, pulmonary hypertension).

Arnaud Bourdin ${ }^{1,2}$, Micaela Romagnoli ${ }^{3}$, Anne Sophie Gamez ${ }^{1}$, Kheira Hireche ${ }^{4}$, Jean Philippe Berthet ${ }^{4}$, Jean Pierre Mallet ${ }^{1}$, Isabelle Vachier ${ }^{1}$, Stefano Nava $^{5}$, Paul Reynaud ${ }^{1}$, Nicolas Molinari ${ }^{6,7}$ and Carey Suehs $\oplus^{1,7}$

${ }^{1}$ Dept of Respiratory Diseases, Univ Montpellier, CHU Montpellier, Montpellier, France. ${ }^{2}$ PhyMedExp, Univ Montpellier, CNRS, INSERM, CHU Montpellier, Montpellier, France. ${ }^{3}$ Pulmonology Unit, Ospedale Ca' Foncello, AULSS2 Marca Trevigiana, Treviso, Italy. ${ }^{4}$ Dept of Cardiac, Thoracic and Vascular Surgery, Univ Montpellier, CHU Montpellier, Montpellier, France. ${ }^{5}$ Dept of Pulmonology, Univ Bologna, S. Orsola-Malpighi Hospital, Bologna, Italy. ${ }^{6} \mathrm{IMAG}$, CNRS, Univ Montpellier, CHU Montpellier, Montpellier, France. ${ }^{7}$ Dept of Medical Information, Univ Montpellier, CHU Montpellier, Montpellier, France.

Correspondence: Carey Suehs, Dept of Respiratory Diseases, Hôpital Arnaud de Villeneuve, 371 Av. du Doyen Gaston Giraud, F-34090 Montpellier, France. E-mail: careysuehs@protonmail.com

Received: 16 Dec 2019 | Accepted after revision: 7 April 2020

Conflict of interest: A. Bourdin reports grants, personal fees for advisory board work, non-financial support for meeting attendance and has been investigator for AstraZeneca, GSK and Boehringer Ingelheim, personal fees for advisory board work, non-financial support for meeting attendance and has been investigator for Novartis, Chiesi Farmaceuticals and Actelion, personal fees for advisory board work and has been investigator for Teva, Regeneron and Roche, and has been investigator for Gilead, outside the submitted work. M. Romagnoli reports grants and personal fees from AstraZeneca, GSK, Roche and Novartis, grants from Boehringer, Menarini, Chiesi and Alfasigma, personal fees from Guidotti, outside the submitted work. A.S. Gamez has nothing to disclose. K. Hireche has nothing to disclose. J.P. Berthet has nothing to disclose. J.P. Mallet has nothing to disclose. I. Vachier has nothing to disclose. S. Nava has nothing to disclose. P. Reynaud has nothing to disclose. N. Molinari has nothing to disclose. C. Suehs has nothing to disclose.

\section{References}

1 Pannu J, Roller LJ, Maldonado F, et al. Transbronchial cryobiopsy for diffuse parenchymal lung disease: 30 - and 90-day mortality. Eur Respir J 2019; 54: 1900337.

2 Hetzel J, Maldonado F, Ravaglia C, et al. Transbronchial cryobiopsies for the diagnosis of diffuse parenchymal lung diseases: expert statement from the Cryobiopsy Working Group on safety and utility and a call for standardization of the procedure. Respiration 2018; 95: 188-200.

3 Lentz RJ, Argento AC, Colby TV, et al. Transbronchial cryobiopsy for diffuse parenchymal lung disease: a state-of-the-art review of procedural techniques, current evidence, and future challenges. J Thorac Dis 2017; 9: 2186-2203.

4 DiBardino DM, Haas AR, Lanfranco AR, et al. High complication rate after introduction of transbronchial cryobiopsy into clinical practice at an academic medical center. Ann Am Thorac Soc 2017; 14: 851-857.

5 Romagnoli M, Colby TV, Berthet J-P, et al. Poor concordance between sequential transbronchial lung cryobiopsy and surgical lung biopsy in the diagnosis of diffuse interstitial lung diseases. Am J Respir Crit Care Med 2019; 199: 1249-1256.

6 Troy LK, Grainge C, Corte TJ, et al. Diagnostic accuracy of transbronchial lung cryobiopsy for interstitial lung disease diagnosis (COLDICE): a prospective, comparative study. Lancet Respir Med 2020; 8: 171-181. 